FACTA UNIVERSITATIS (NIŠ)

Ser. Math. Inform. Vol. 35, No 4 (2020), 1059-1078

https://doi.org/10.22190/FUMI2004059V

\title{
EIGHTY ONE RICCI-TYPE IDENTITIES *
}

\author{
Nenad O. Vesić
}

(C) 2020 by University of Niš, Serbia | Creative Commons Licence: CC BY-NC-ND

Abstract. In this manuscript, the identities of Ricci Type with respect to a nonsymmetric affine connection space are obtained and simplified. The components of commutation formulae are discussed.

Key words: covariant derivative; identities of Ricci Type; commutation formula.

\section{Introduction}

An $N$-dimensional manifold $\mathcal{M}_{N}$ equipped with an affine connection with torsion $\nabla$ is the non-symmetric affine connection space $\mathbb{G A}_{N}$ (see L. P. Eisenhart [1], S. M. Minčić [4-6,6-8]), M. S. Stanković [13], Lj. S. Velimirović $[10,11]$, M. Lj. Zlatanović $[13,14]$, M. Z. Petrović [9-11]. The non-symmetric affine connection spaces are subjects of research for many other authors but our aim is to examine some basic facts about these spaces in this paper.

The affine connection coefficients for the affine connection $\nabla$ are $L_{j k}^{i}$. These coefficients are non-symmetric by indices $j$ and $k$. Hence, their symmetric and anti-symmetric parts are defined as

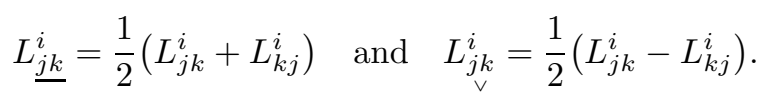

Four kinds of covariant derivatives with respect to the non-symmetric affine connection $\nabla$ are defined. Coordinately, these four types (for a tensor $a_{j}^{i}$ of the type $(1,1))$ are $[4-11,13,14]$

Received October 28, 2019; accepted June 24, 2020

2020 Mathematics Subject Classification. Primary 53B05; Secondary 15A72, 15A03

* This work was supported by the Serbian Ministry of Education, Science and Technological Development through Mathematical Institute of the Serbian Academy of Sciences and Arts. 


$$
\begin{array}{ll}
a_{j \mid k}^{i}=a_{j, k}^{i}+L_{\alpha k}^{i} a_{j}^{\alpha}-L_{j k}^{\alpha} a_{\alpha}^{i}, & a_{j \mid k}^{i}=a_{j, k}^{i}+L_{k \alpha}^{i} a_{j}^{\alpha}-L_{k j}^{\alpha} a_{\alpha}^{i}, \\
a_{j \mid k}^{i}=a_{j, k}^{i}+L_{\alpha k}^{i} a_{j}^{\alpha}-L_{k j}^{\alpha} a_{\alpha}^{i}, & a_{j \mid k}^{i}=a_{j, k}^{i}+L_{k \alpha}^{i} a_{j}^{\alpha}-L_{j k}^{\alpha} a_{\alpha}^{i} .
\end{array}
$$

In the case of $L_{j k}^{i}=0$, the four kinds of covariant derivatives (1.2) reduce to one kind $[2,12]$

$$
a_{j \mid k}^{i}=a_{\substack{j \mid k \\ 0}}^{i}=a_{j, k}^{i}+L_{\underline{\alpha k}}^{i} a_{j}^{\alpha}-L_{\underline{j k}}^{\alpha} a_{\alpha}^{i},
$$

Proposition 1.1. The fourth kind of the covariant derivative expressed in (1.2) and the covariant derivative with respect to the symmetric affine connection given by (1.3) satisfy the equalities

$$
\begin{aligned}
& a_{\substack{j \mid k \\
4}}^{i}=a_{j \mid k}^{i}+a_{\substack{j \mid k \\
1}}^{i}-a_{j \mid k}^{i}, \\
& a_{j \mid k}^{i}=\frac{1}{2} a_{j \mid k}^{i}+\frac{1}{2} a_{j \mid k}^{i} .
\end{aligned}
$$

If $L_{\vee}^{i} \not \equiv 0$, the geometrical objects $a_{j \mid k}^{i}, a_{j \mid k}^{i}, a_{j \mid k}^{i}$ are linearly independent.

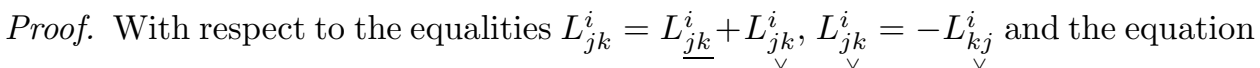
(1.3), one gets

$$
\begin{aligned}
& a_{j \mid k}^{i}=a_{j \mid k}^{i}+L_{\alpha k}^{i} a_{j}^{\alpha}-L_{j k}^{\alpha} a_{\alpha}^{i}, \quad a_{j \mid k}^{i}=a_{j \mid k}^{i}-L_{\alpha k}^{i} a_{j}^{\alpha}+L_{j k}^{\alpha} a_{\alpha}^{i}, \\
& a_{j \mid k}^{i}=a_{j \mid k}^{i}+L_{\alpha k}^{i} a_{j}^{\alpha}+L_{\substack{j k \\
\alpha}}^{i} a_{\alpha}^{i}, \quad a_{j \mid k}^{i}=a_{j \mid k}^{i}-L_{\alpha k}^{i} a_{j}^{\alpha}-L_{j k}^{\alpha} a_{\alpha}^{i},
\end{aligned}
$$

From the expressions (1.5), one obtains $[9,10]$

$$
a_{j \mid k}^{i}=a_{j \mid k}^{i}+a_{j \mid k}^{i}-a_{j \mid k}^{i} \quad \text { and } \quad a_{j \mid k}^{i}=\frac{1}{2} a_{j \mid k}^{i}+\frac{1}{2} a_{j \mid k}^{i}
$$

which proves the first part of this proposition.

Furthermore, the geometrical objects $a_{j \mid k}^{i}, a_{j \mid k}^{i}, a_{j \mid k}^{i}$ expressed as in the equation (1.5) may be considered as the vectors $v_{1}=(1,1,-1), v_{2}=(1,-1,1), v_{3}=(1,1,1)$. These vectors are linearly independent, which completes the proof for this proposition. 
Curvatures of the space $\mathbb{G A}_{N}$ are $a_{j_{1 \mid m} \mid{ }_{w_{1}} n}^{i}-a_{j|n| m \mid m}^{i} \underset{v_{2} w_{2}}{i}$, for $v_{1}, v_{2}, w_{1}, w_{2} \in$ $\{0,1,2,3,4\}$. We will study the curvatures of the space $\mathbb{G A}_{N}$ obtained with respect to the first three kinds of covariant derivatives (1.2) in this paper.

Our purpose is to coordinately express the curvatures of the space $\mathbb{G A}_{N}$ with respect to first three kinds of covariant derivatives (1.2) in this paper. We will obtain the coordinates of the differences $a_{j_{v_{1}|m| n} i_{w_{1}} n}^{i}-a_{\substack{j|n| m \\ v_{2} w_{2}}}^{i}$, for $v_{1}, v_{2}, w_{1}, w_{2} \in$ $\{1,2,3\}$. The pseudocurvature tensors as possible components of these differences will be discussed. The number of linearly independent geometrical objects $a_{j \backslash m \mid w_{w_{1}} n}^{i}-a_{j \backslash n\left|v_{2}\right| m}^{i}, v_{1}, v_{2}, w_{1}, w_{2} \in\{1,2,3\}$, will be obtained. At the end of the paper, we will list all of the commutation formulae with respect to $a_{\substack{v_{1} m \mid w_{1} \\ i}}-a_{j_{v_{2}|n| w_{2}}}^{i}$, $v_{1}, v_{2}, w_{1}, w_{2} \in\{1,2,3\}$.

\section{Identities of Ricci type}

With respect to the equations $(1.3,1.5)$, one gets

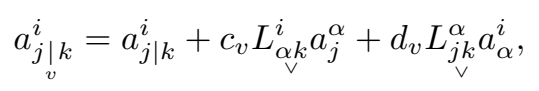

for $v=0, \ldots, 4$ and $c_{0}=0, c_{1}=1, c_{2}=-1, c_{3}=1, c_{4}=-1, d_{0}=0, d_{1}=-1$, $d_{2}=1, d_{3}=1, d_{4}=-1$.

Moreover, it holds the equation

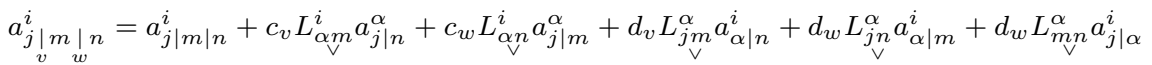

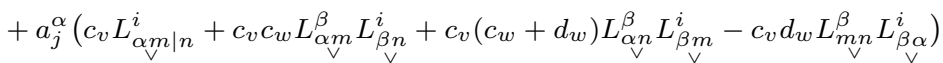

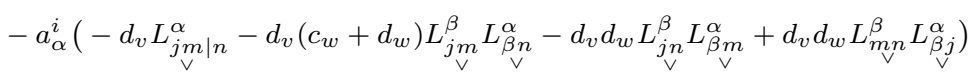

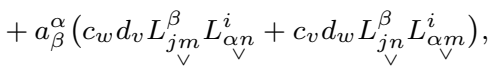

for $v, w \in\{0,1,2,3,4\}$.

The next theorem holds. 
Theorem 2.1. First Ricci-Type Identities Theorem The family of identities of the Ricci Type with respect to a non-symmetric affine connection $\nabla$ is

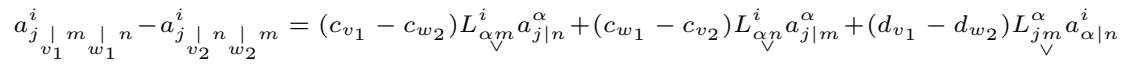

$$
\begin{aligned}
& +\left(d_{w_{1}}-d_{v_{2}}\right) L_{j^{n}}^{\alpha} a_{\alpha \mid m}^{i}+\left(d_{w_{1}}+d_{w_{2}}\right) L_{m^{n} n}^{\alpha} a_{j \mid \alpha}^{i} \\
& +a_{j}^{\alpha}\left\{R_{\alpha m n}^{i}+c_{v_{1}} L_{\alpha \underset{V}{ } \mid n}^{i}-c_{v_{2}} L_{\underset{\vee}{\alpha n} \mid m}^{i}\right.
\end{aligned}
$$

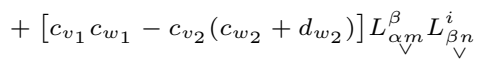

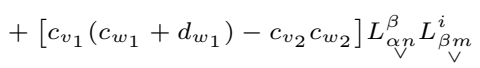

$$
\begin{aligned}
& \left.-\left(c_{v_{1}} d_{w_{1}}+c_{v_{2}} d_{w_{2}}\right) L_{m_{\mathrm{n}}}^{\beta} L_{\beta \alpha}^{i}\right\}
\end{aligned}
$$

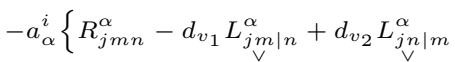

$$
\begin{aligned}
& -\left[d_{v_{1}}\left(c_{w_{1}}+d_{w_{1}}\right)-d_{v_{2}} d_{w_{2}}\right] L_{j m}^{\beta} L_{j^{\prime} n}^{\alpha}
\end{aligned}
$$

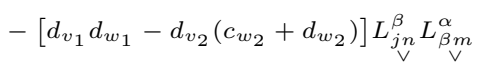

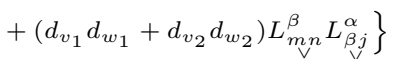

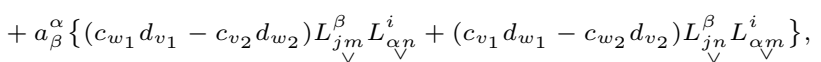

for $v_{1}, v_{2}, w_{1}, w_{2} \in\{0,1,2,3,4\}$.

From this theorem, we obtain that just tensors are components of the curvatures for the space $\mathbb{G A}_{N}$.

The rank of the matrix of the type $81 \times 19$ whose rows are composed of the elements

$c_{v_{1}}-c_{w_{2}}, \quad c_{w_{1}}-c_{v_{2}}, \quad d_{v_{1}}-d_{w_{2}}, \quad d_{w_{1}}-d_{v_{2}}, \quad d_{w_{1}}+d_{w_{2}}$,

$1, \quad c_{v_{1}}, \quad-c_{v_{2}}, \quad c_{v_{1}} c_{w_{1}}-c_{v_{2}}\left(c_{w_{2}}+d_{w_{2}}\right), \quad c_{v_{1}}\left(c_{w_{1}}+d_{w_{1}}\right)-c_{v_{2}} c_{w_{2}}, \quad-\left(c_{v_{1}} d_{w_{1}}+c_{v_{2}} d_{w_{2}}\right)$,

$-1, \quad d_{v_{1}}, \quad-d_{v_{2}}, \quad d_{v_{1}}\left(c_{w_{1}}+d_{w_{1}}\right)-d_{v_{2}} d_{w_{2}}, \quad d_{v_{1}} d_{w_{1}}-d_{v_{2}}\left(c_{w_{2}}+d_{w_{2}}\right), \quad-\left(d_{v_{1}} d_{w_{1}}+d_{v_{2}} d_{w_{2}}\right)$, $c_{w_{1}} d_{v_{1}}-c_{v_{2}} d_{w_{2}}, \quad c_{v_{1}} d_{w_{1}}-c_{w_{2}} d_{v_{2}}$,

for $v_{1}, v_{2}, w_{1}, w_{2} \in\{1,2,3\}$, is 15 .

In this way, we proved the next theorem.

Theorem 2.2. $1-2-3$-Commutation Formulae Theorem Fifteen of the geometrical objects $a_{j \mid m}^{i} \underset{v_{1} \mid w_{1} n}{i}-a_{j|n|}^{i} v_{v_{2} w_{2}}$, for $v_{1}, v_{2}, w_{1}, w_{2} \in\{1,2,3\}$, are linearly independent.

One may check that the geometrical objects 


$$
\begin{aligned}
& \mathcal{B}_{(1), j m n}^{i}=a_{j|1| 1}^{i}-a_{j|1|}^{i}
\end{aligned}
$$

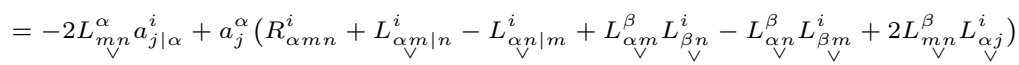

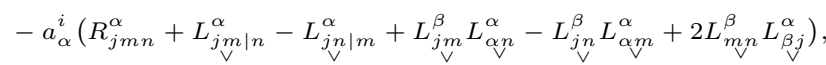

$$
\begin{aligned}
& \mathcal{B}_{(2) . j m n}^{i}=a_{j|m| n}^{i}-a_{j|2| m \mid m}^{i}
\end{aligned}
$$

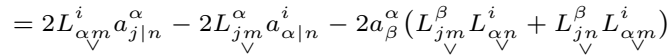

$$
\begin{aligned}
& +a_{j}^{\alpha}\left(R_{\alpha m n}^{i}+L_{\alpha m \mid n}^{i}-L_{\alpha n \mid m}^{i}+L_{\alpha m}^{\beta} L_{\beta n}^{i}+L_{\substack{\alpha n}}^{i} L_{\beta m}^{i}\right)
\end{aligned}
$$

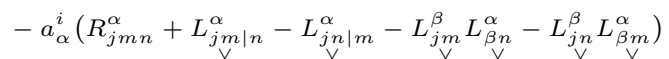

$$
\begin{aligned}
& \mathcal{B}_{(3) \cdot j m n}^{i}=a_{j|m| 1}^{i}-a_{j|3| m}^{i}
\end{aligned}
$$

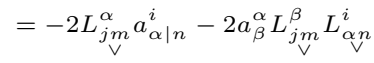

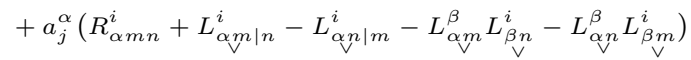

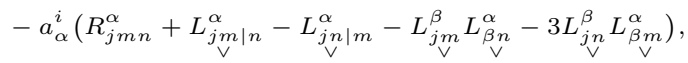

$$
\begin{aligned}
& \mathcal{B}_{(4) \cdot j m n}^{i}=a_{j|m| 1}^{i}-a_{j|2|}^{i}{ }_{1}^{i}
\end{aligned}
$$

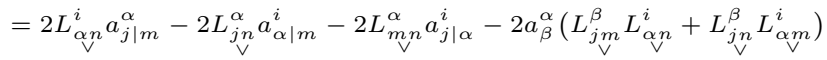

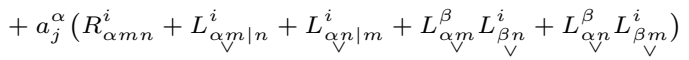

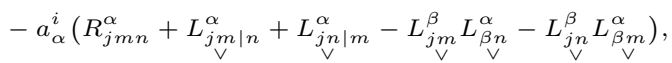

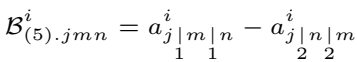

$$
\begin{aligned}
& =2 L_{\alpha m}^{i} a_{j \mid n}^{\alpha}+2 L_{\alpha,}^{i} a_{j \mid m}^{\alpha}-2 L_{j m}^{\alpha} a_{\alpha \mid n}^{i}-2 L_{j n}^{\alpha} a_{\alpha \mid m}^{i}
\end{aligned}
$$

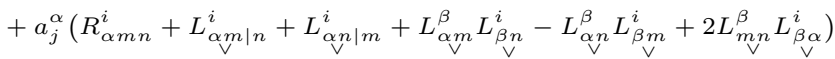

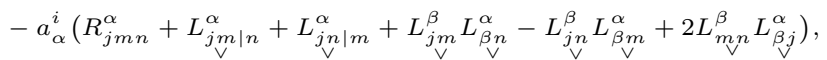

$$
\begin{aligned}
& \mathcal{B}_{(6) . j m n}^{i}=a_{j|m| n}^{i}-a_{\substack{j|n| m \\
1}}^{i} \\
& =2 L_{\alpha, n}^{i} a_{j \mid m}^{\alpha}-2 L_{j m}^{\alpha} a_{\alpha \mid n}^{i}-2 L_{j n}^{\alpha} a_{\alpha \mid m}^{i}-2 a_{\beta}^{\alpha} L_{j n}^{\beta} L_{\alpha m}^{i}
\end{aligned}
$$

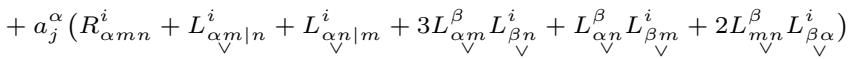

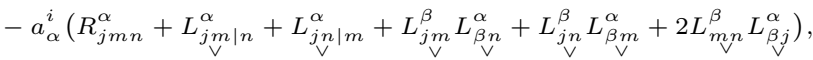


1064

N. O. Vesić

(2.10)

$$
\begin{aligned}
& \mathcal{B}_{(7) \cdot j m n}^{i}=a_{\substack{j|m| n \\
1}}^{i}-a_{\substack{j|n| m \\
3 \mid 1}}^{i}
\end{aligned}
$$

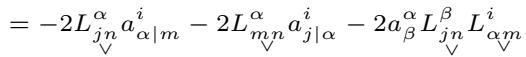

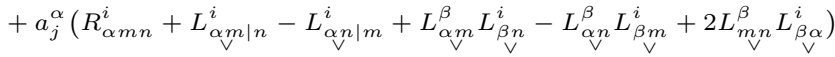

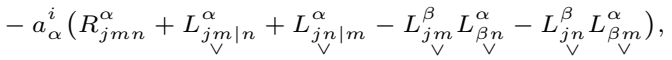

$$
\begin{aligned}
& \mathcal{B}_{(8) \cdot j m n}^{i}=a_{\substack{j|m| n \\
1}}^{i}-a_{\substack{j|n| m \\
3}}^{i}
\end{aligned}
$$

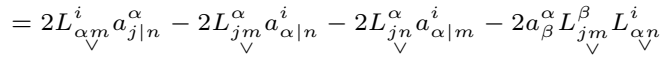

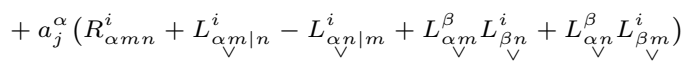

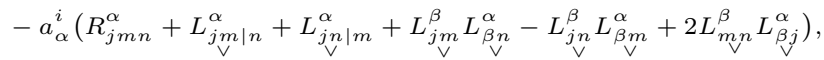

$$
\begin{aligned}
& \mathcal{B}_{(9) \cdot j m n}^{i}=a_{j|m| n}^{i}-a_{\substack{j|n| m \\
3}}^{i}
\end{aligned}
$$

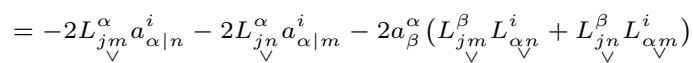

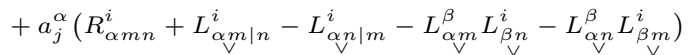

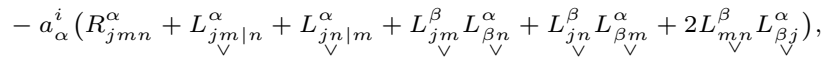

$$
\begin{aligned}
& \mathcal{B}_{(10) \cdot j m n}^{i}=a_{j|m| 2}^{i}-a_{j|1| m}^{i}
\end{aligned}
$$

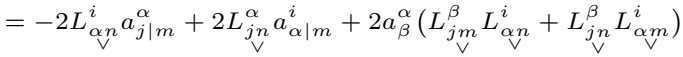

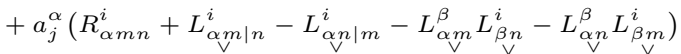

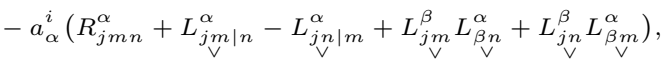

$$
\begin{aligned}
& \mathcal{B}_{(11), j m n}^{i}=a_{j|m| n}^{i}-a_{j|1| m \mid m}^{i}
\end{aligned}
$$

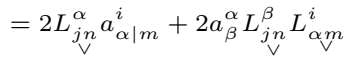

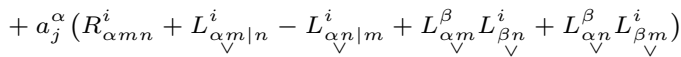

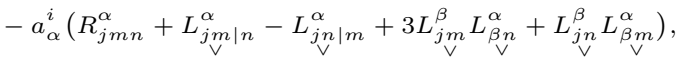

$$
\begin{aligned}
& \mathcal{B}_{(12) . j m n}^{i}=a_{j|m| n}^{i}-a_{j|n| m}^{i}
\end{aligned}
$$

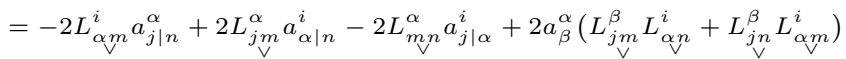

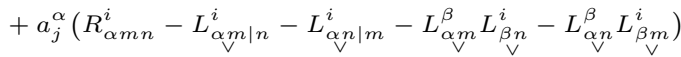

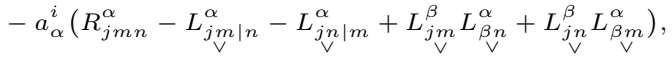




$$
\begin{aligned}
& \mathcal{B}_{(13) . j m n}^{i}=a_{\substack{j|m| n \\
2}}^{i}-a_{j|n| m \mid m}^{i} \\
& =-2 L_{\alpha, m}^{i} a_{j \mid n}^{\alpha}-2 L_{\alpha, n}^{i} a_{j \mid m}^{\alpha}+2 L_{j m}^{\alpha} a_{\alpha \mid n}^{i}+2 L_{j n}^{\alpha} a_{\alpha \mid m}^{i}
\end{aligned}
$$

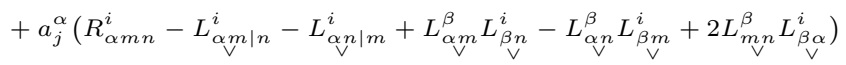

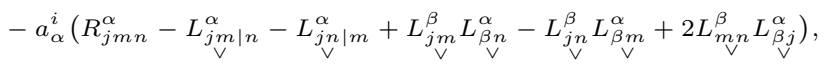

$$
\begin{aligned}
& \mathcal{B}_{(14) . j m n}^{i}=\underset{\substack{2 \\
a_{3}|m| n}}{i}-a_{j|1| m}^{i}
\end{aligned}
$$

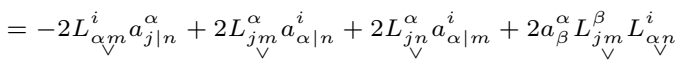

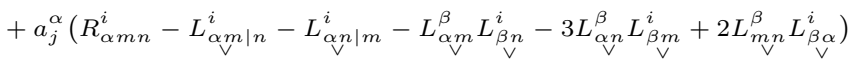

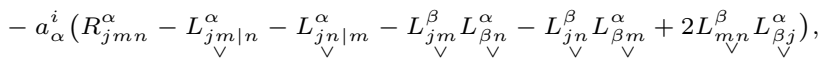

$$
\begin{aligned}
& \mathcal{B}_{(15) \cdot j m n}^{i}=a_{\substack{j|m| n \\
i}}^{i}-a_{j|1|}^{i} \\
& =a_{j|m| n}^{i}-a_{j|n| m}^{i}
\end{aligned}
$$

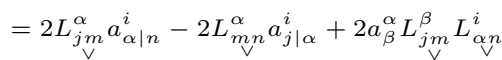

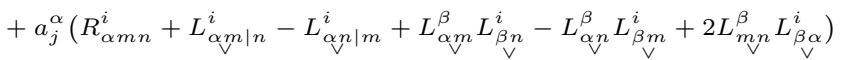

$$
\begin{aligned}
& -a_{\alpha}^{i}\left(R_{j m n}^{\alpha}-L_{\vee j \mid n}^{\alpha}-L_{\vee}^{\alpha} \mid m+L_{j m}^{\beta} L_{\beta n}^{\alpha}+L_{j n}^{\beta} L_{\beta m}^{\alpha}\right),
\end{aligned}
$$

are a base of the vector spaces generated by the differences $a_{j \mid m}^{i} \underset{v_{1} \mid w_{w_{1}} n}{ }-a_{j|n| m \mid m}^{i}$, $v_{1}, v_{2}, w_{1}, w_{2} \in\{1,2,3\}$.

With respect to the equation (2.3), we obtain that many curvature tensors but no one curvature pseudotensor may be obtained with respect to the identities of Ricci Type presented in the First Ricci-Type Identities Theorem.

Vice versa, any linear combination of the geometrical objects $b_{(k) j m n}^{i}$, $k=1, \ldots, 16$, corresponds to infinitely many linear combinations of the differences $a_{j|m| n}^{i} \underset{v_{1} w_{1}}{i}-a_{j|n| m}^{i} \underset{v_{2} w_{2}}{i}, v_{1}, v_{2}, w_{1}, w_{2} \in\{0,1,2,3,4\}$.

To obtain curvature pseudotensors for the space $\mathbb{G A}_{N}$, we need to consider the base $\left(c_{(k) j m n}^{i}\right)=\left(b_{(k) j m n}^{i}+\mathcal{L}_{(k) j m n}^{i}\right), k=1, \ldots, 16$, where the geometrical objects $\mathcal{L}_{(k) j m n}^{i}$ are linear combinations of the products $L_{\underline{\alpha n}}^{i} L_{j m}^{\alpha}, L_{\underline{\alpha m}}^{i} L_{\vee}^{\alpha n}, L_{\underline{\alpha}}^{i} L_{\underset{\vee}{m n}}^{\alpha}$, $L_{\underset{\vee}{\alpha n}}^{i} L_{\underline{j m}}^{\alpha}, L_{\alpha \underset{\vee}{i}}^{i} L_{\underline{j n}}^{\alpha}, L_{\alpha j}^{i} L_{\underline{m n}}^{\alpha}$.

Any linear combination of the geometrical objects $c_{(k) j m n}^{i}$ does not correspond

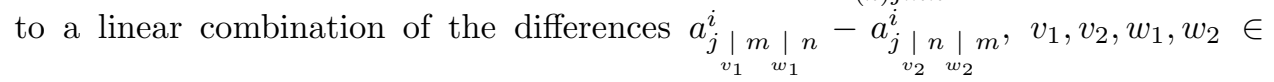
$\{0,1,2,3,4\}$.

For this reason, the geometrical objects $b_{(k) j m n}^{i}$ are components of a base for

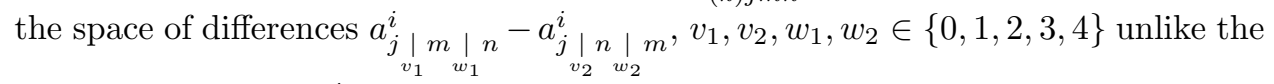
geometrical objects $c_{(k) j m n}^{i}$. 
Remark 2.1. Any identity of Ricci Type where the curvature pseudotensors of the space $\mathbb{G A}_{N}$ are obtained may be simplified and reduced to the form (2.3).

\subsection{Eighty one Ricci-Type identities}

With respect to the First Ricci-Type Identities Theorem, and for $v_{1}, v_{2}, w_{1}, w_{2} \in$ $\{1,2,3\}$, we obtain the next identities of Ricci Type.

$$
\begin{aligned}
& a_{j|m| n}^{i}-a_{j|1, n| m}^{i}=-2 L_{\underset{1}{m} n}^{\alpha} a_{j \mid \alpha}^{i}
\end{aligned}
$$

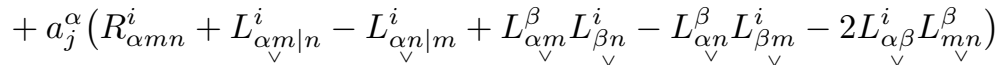

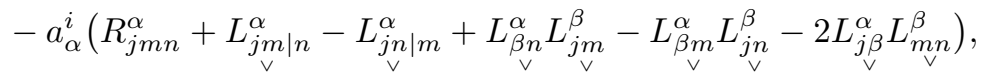

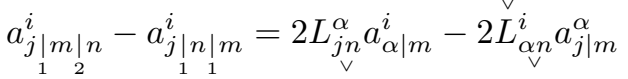

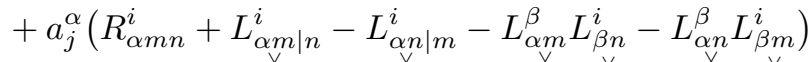

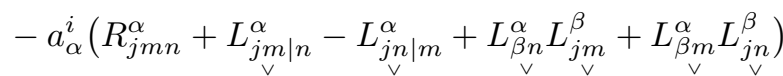

$$
\begin{aligned}
& +2 a_{\beta}^{\alpha}\left(L_{\alpha \underset{\vee}{ }}^{i} L_{j n}^{\beta}+L_{\alpha \vee}^{i} L_{j m}^{\beta}\right)
\end{aligned}
$$

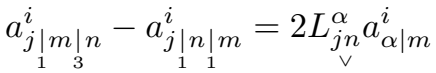

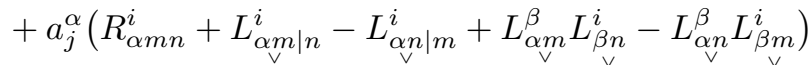

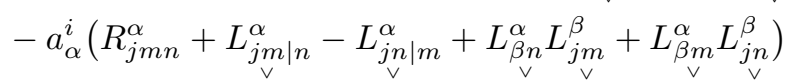

$$
\begin{aligned}
& +2 a_{\beta}^{\alpha} L_{\alpha m}^{i} L_{j n}^{\beta}
\end{aligned}
$$

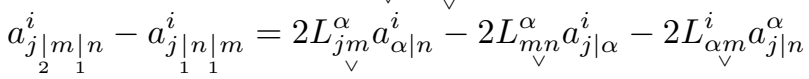

$$
\begin{aligned}
& +a_{j}^{\alpha}\left(R_{\alpha m n}^{i}-L_{\alpha \underset{\vee}{ } \mid n}^{i}-L_{\underset{\vee}{\alpha n \mid m}}^{i}-L_{\alpha m}^{\beta} L_{\beta n}^{i}-L_{\substack{\alpha n \\
\beta}}^{\beta} L_{\beta m}^{i}\right)
\end{aligned}
$$

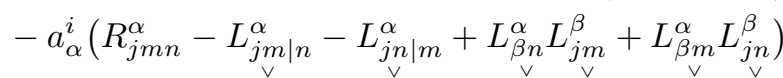

$$
\begin{aligned}
& +2 a_{\beta}^{\alpha}\left(L_{\alpha n}^{i} L_{j m}^{\beta}+L_{\alpha m}^{i} L_{j n}^{\beta}\right),
\end{aligned}
$$

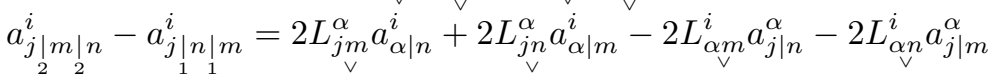

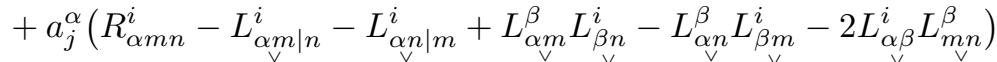

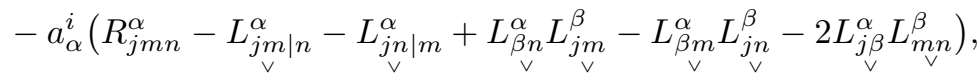




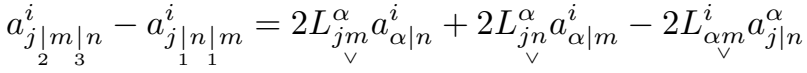

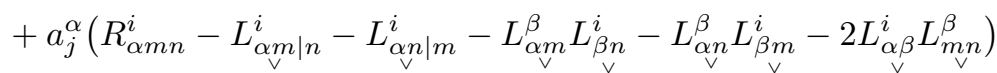

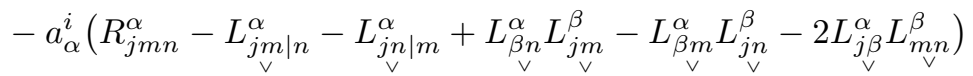

$$
\begin{aligned}
& +2 a_{\beta}^{\alpha} L_{\alpha n}^{i} L_{j m}^{\beta}
\end{aligned}
$$

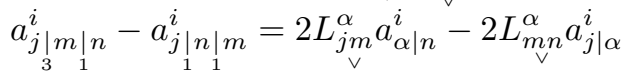

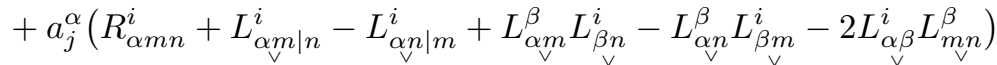

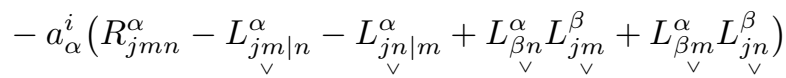

$$
\begin{aligned}
& +2 a_{\beta}^{\alpha} L_{\alpha n}^{i} L_{j m}^{\beta}
\end{aligned}
$$

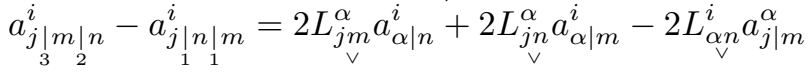

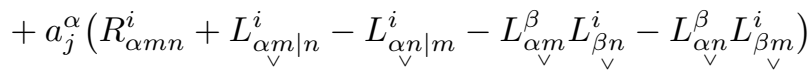

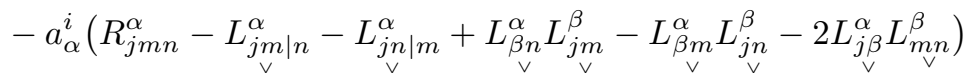

$$
\begin{aligned}
& +2 a_{\beta}^{\alpha} L_{\alpha m}^{i} L_{j n}^{\beta}
\end{aligned}
$$

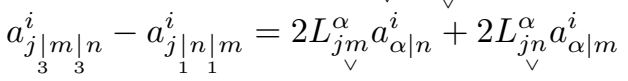

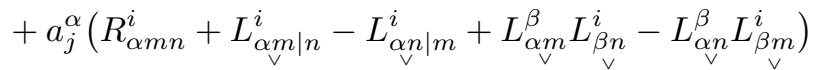

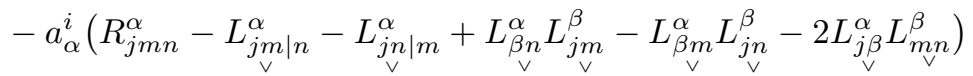

$$
\begin{aligned}
& +2 a_{\beta}^{\alpha}\left(L_{\underset{\vee}{\alpha n}}^{i} L_{j m}^{\beta}+L_{\alpha m}^{i} L_{j n}^{\beta}\right) \text {, }
\end{aligned}
$$

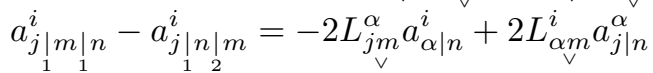

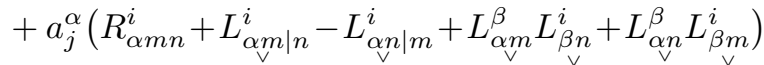

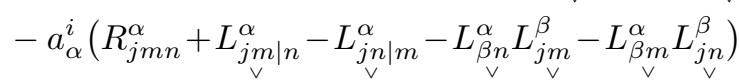

$$
\begin{aligned}
& -2 a_{\beta}^{\alpha}\left(L_{\alpha m}^{i} L_{j n}^{\beta}+L_{\alpha \vee}^{i} L_{j m}^{\beta}\right) \text {, }
\end{aligned}
$$

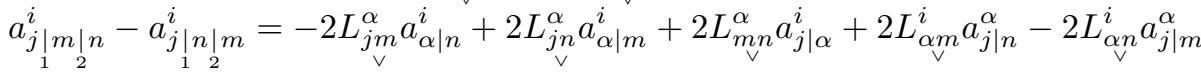

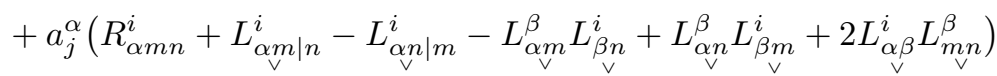

$$
\begin{aligned}
& -a_{\alpha}^{i}\left(R_{j m n}^{\alpha}+L_{j m \mid n}^{\alpha}-L_{j n \mid m}^{\alpha}-L_{\beta n}^{\alpha} L_{j m}^{\beta}+L_{\beta m}^{\alpha} L_{j n}^{\beta}+2 L_{j \beta}^{\alpha} L_{m_{\vee} n}^{\beta}\right),
\end{aligned}
$$

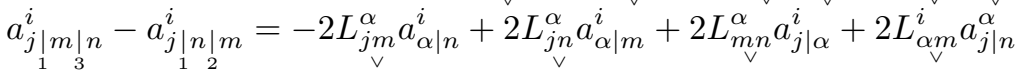

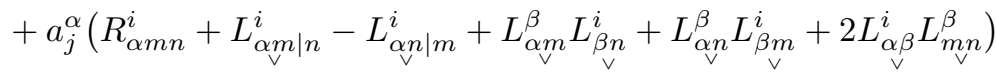

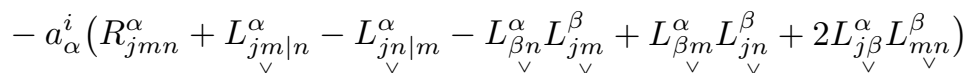

$$
\begin{aligned}
& -2 a_{\beta}^{\alpha} L_{\underset{\vee}{\alpha n}}^{i} L_{j m}^{\beta}
\end{aligned}
$$




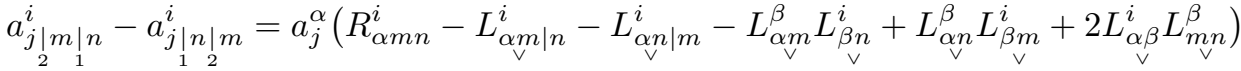

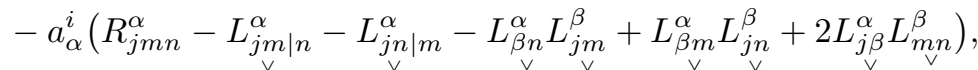

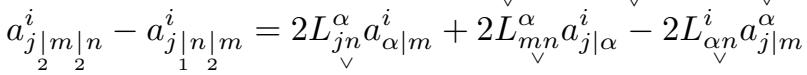

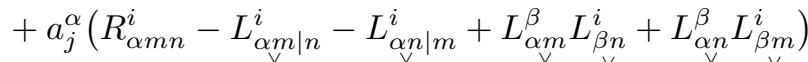

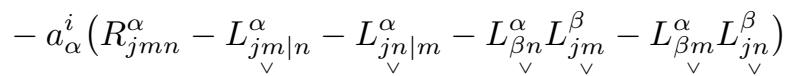

$$
\begin{aligned}
& -2 a_{\beta}^{\alpha}\left(L_{\alpha m}^{i} L_{j n}^{\beta}+L_{\alpha \vee}^{i} L_{j m}^{\beta}\right) \text {, } \\
& a_{\substack{|m| m \mid n \\
i}}^{i}-a_{\substack{|, n| m \\
i}}^{i}=2 L_{\substack{j n \\
\alpha}}^{\alpha} a_{\alpha \mid m}^{i}+2 L_{\underset{\vee}{m} n}^{\alpha} a_{j \mid \alpha}^{i} \\
& +a_{j}^{\alpha}\left(R_{\alpha m n}^{i}-L_{\underset{\vee}{\alpha m \mid n}}^{i}-L_{\underset{\vee}{\alpha n \mid m}}^{i}-L_{\underset{\vee}{\alpha}}^{\beta} L_{\beta n}^{i}+L_{\underset{\vee}{\alpha n}}^{\beta} L_{\beta m}^{i}\right)
\end{aligned}
$$

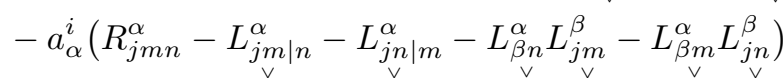

$$
\begin{aligned}
& -2 a_{\beta}^{\alpha} L_{\alpha m}^{i} L_{j n}^{\beta}
\end{aligned}
$$

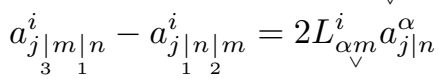

$$
\begin{aligned}
& +a_{j}^{\alpha}\left(R_{\alpha m n}^{i}+L_{\alpha m \mid n}^{i}-L_{\alpha \vee \mid m}^{i}+L_{\alpha m}^{\beta} L_{\beta n}^{i}+L_{\alpha n}^{\beta} L_{\beta m}^{i}\right)
\end{aligned}
$$

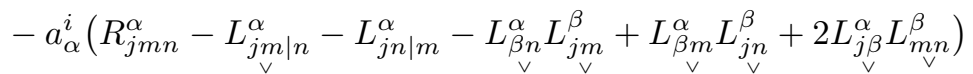

$$
\begin{aligned}
& -2 a_{\beta}^{\alpha} L_{\alpha m}^{i} L_{j n}^{\beta}
\end{aligned}
$$

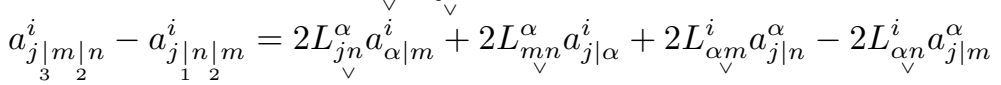

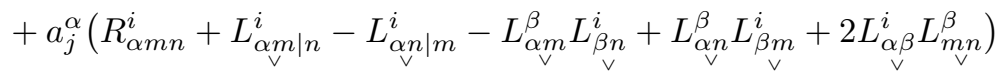

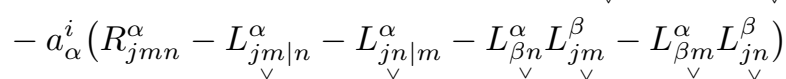

$$
\begin{aligned}
& -2 a_{\beta}^{\alpha} L_{\alpha \eta}^{i} L_{j m}^{\beta}
\end{aligned}
$$

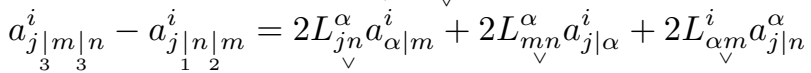

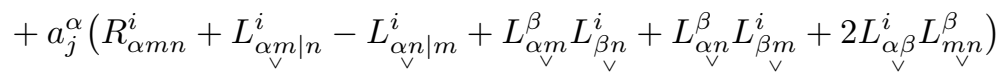

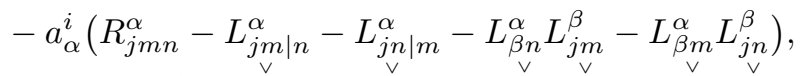

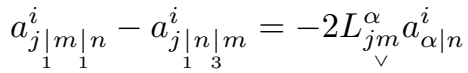

$$
\begin{aligned}
& +a_{j}^{\alpha}\left(R_{\alpha m n}^{i}+L_{\underset{\vee}{\alpha m \mid n}}^{i}-L_{\underset{\vee}{\alpha n \mid m}}^{i}+L_{\underset{\vee}{\alpha m}}^{\beta} L_{\beta n}^{i}-L_{\underset{\vee}{\alpha n}}^{\beta} L_{\beta m}^{i}\right)
\end{aligned}
$$

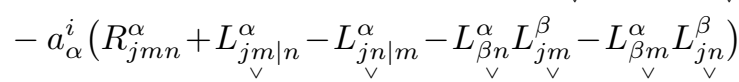

$$
\begin{aligned}
& -2 a_{\beta}^{\alpha} L_{\underset{\vee}{\alpha n}}^{i} L_{j m}^{\beta}
\end{aligned}
$$




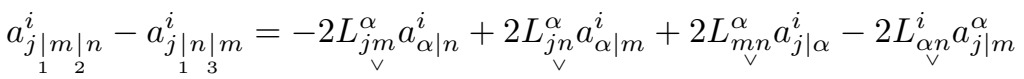

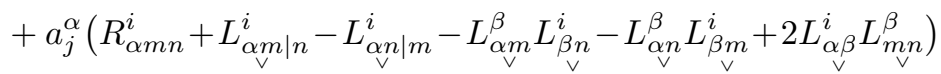

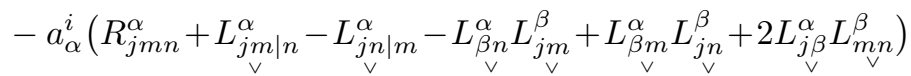

$$
\begin{aligned}
& +2 a_{\beta}^{\alpha} L_{\alpha m}^{i} L_{j n}^{\beta}
\end{aligned}
$$

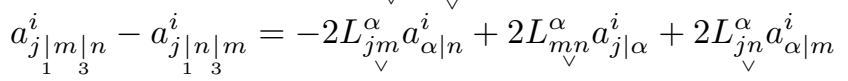

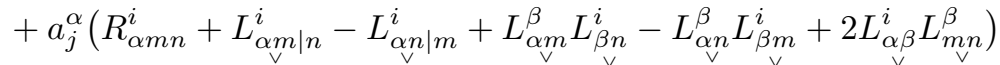

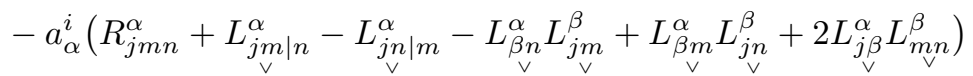

$$
\begin{aligned}
& +2 a_{\beta}^{\alpha}\left(L_{\alpha m}^{i} L_{j n}^{\beta}-L_{\alpha \vee}^{i} L_{j m}^{\beta}\right),
\end{aligned}
$$

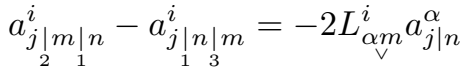

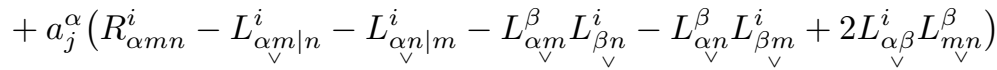

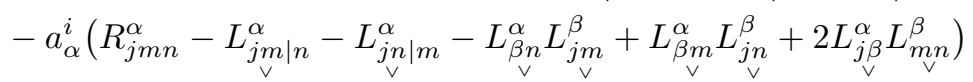

$$
\begin{aligned}
& +2 a_{\beta}^{\alpha} L_{\alpha m}^{i} L_{j n}^{\beta}
\end{aligned}
$$

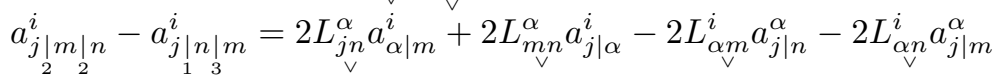

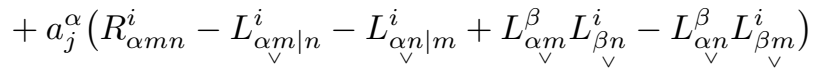

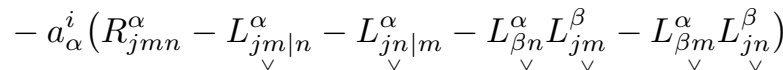

$$
\begin{aligned}
& -2 a_{\beta}^{\alpha} L_{\alpha n}^{i} L_{j m}^{\beta}
\end{aligned}
$$

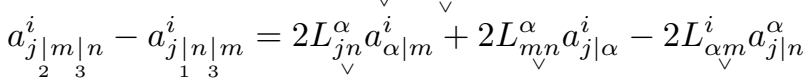

$$
\begin{aligned}
& +a_{j}^{\alpha}\left(R_{\alpha m n}^{i}-L_{\alpha m \mid n}^{i}-L_{\alpha n \mid m}^{i}-L_{\alpha m}^{\beta} L_{\beta n}^{i}-L_{\alpha \vee}^{\beta} L_{\beta m}^{i}\right) \\
& -a_{\alpha}^{i}\left(R_{j m n}^{\alpha}-L_{j m \mid n}^{\alpha}-L_{j n \mid m}^{\alpha}-L_{\beta n}^{\alpha} L_{j m}^{\beta}-L_{\beta m}^{\alpha} L_{j n}^{\beta}\right),
\end{aligned}
$$

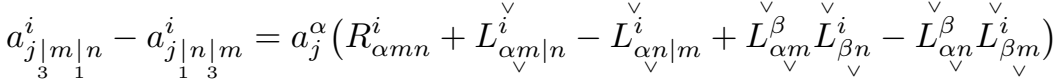

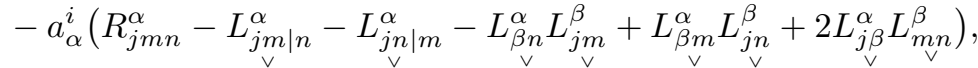

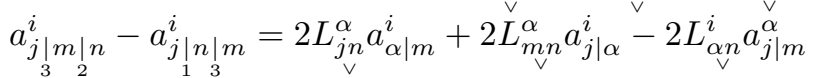

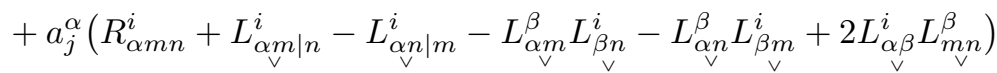

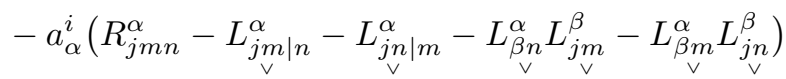

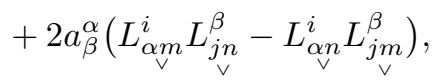




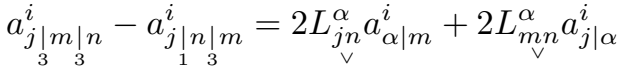

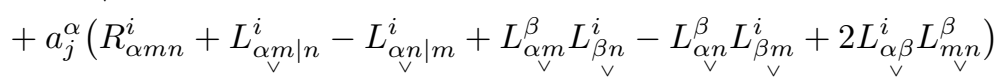

$$
\begin{aligned}
& -a_{\alpha}^{i}\left(R_{j m n}^{\alpha}-L_{j m \mid n}^{\alpha}-L_{j n \mid m}^{\alpha}-L_{\beta \vee}^{\alpha} L_{j m}^{\beta}-L_{\beta m}^{\alpha} L_{j n}^{\beta}\right) \\
& +2 a_{\beta}^{\alpha} L_{\alpha m}^{i} L_{j n}^{\beta},
\end{aligned}
$$

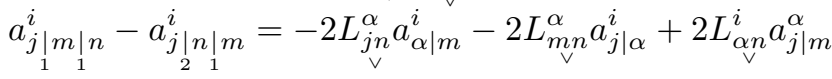

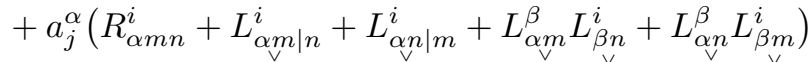

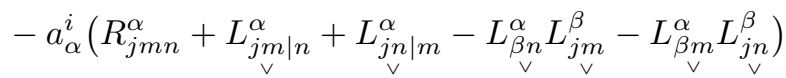

$$
\begin{aligned}
& -2 a_{\beta}^{\alpha}\left(L_{\alpha n}^{i} L_{j m}^{\beta}+L_{\alpha m}^{i} L_{j n}^{\beta}\right),
\end{aligned}
$$

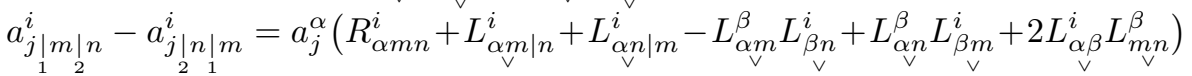

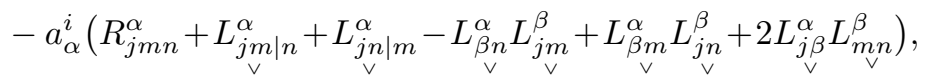

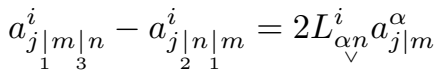

$$
\begin{aligned}
& +a_{j}^{\alpha}\left(R_{\alpha m n}^{i}+L_{\alpha m \mid n}^{i}+L_{\alpha n \mid m}^{i}+L_{\alpha m}^{\beta} L_{\beta n}^{i}+L_{\alpha n}^{\beta} L_{\beta m}^{i}+2 L_{\alpha \beta}^{i} L_{m n}^{\beta}\right) \\
& -a_{\alpha}^{i}\left(R_{j m n}^{\alpha}+L_{j m \mid n}^{\alpha}+L_{j \vee \mid m}^{\alpha}-L_{\beta n}^{\alpha} L_{j m}^{\beta}+L_{\beta m}^{\alpha} L_{j \vee}^{\beta}+2 L_{j \beta}^{\alpha} L_{m_{\vee} n}^{\beta}\right) \\
& -2 a_{\beta}^{\alpha} L_{\alpha \vee}^{i} L_{j m}^{\beta},
\end{aligned}
$$

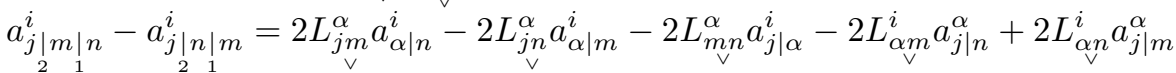

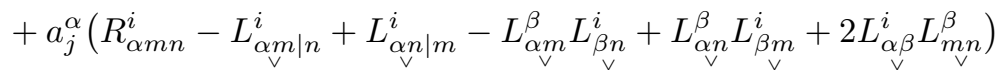

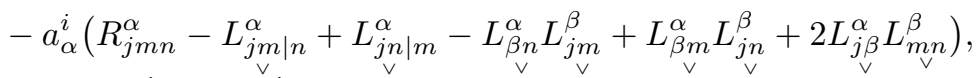

$$
\begin{aligned}
& a_{\substack{|m| m \mid n \\
2}}^{i}-a_{\substack{|, n| m \\
2}}^{i}=2 L_{\substack{j m \\
\alpha \mid n}}^{\alpha} a_{\vee}^{i}-2 L_{\alpha m}^{i} a_{j \mid n}^{\alpha} \\
& +a_{j}^{\alpha}\left(R_{\alpha m n}^{i}-L_{\alpha m \mid n}^{i}+L_{\alpha n \mid m}^{i}+L_{\alpha m}^{\beta} L_{\beta n}^{i}+L_{\alpha n}^{\beta} L_{\beta m}^{i}\right)
\end{aligned}
$$

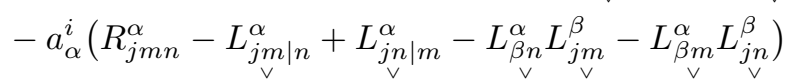

$$
\begin{aligned}
& -2 a_{\beta}^{\alpha}\left(L_{\alpha m}^{i} L_{j n}^{\beta}+L_{\alpha n}^{i} L_{j m}^{\beta}\right),
\end{aligned}
$$

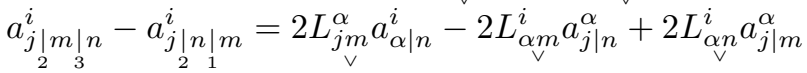

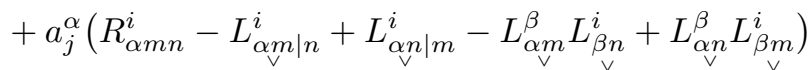

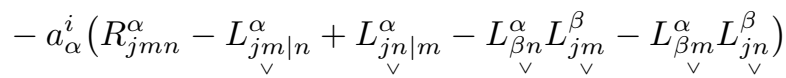

$$
\begin{aligned}
& -2 a_{\beta}^{\alpha} L_{\alpha \underset{v}{ }}^{i} L_{j n}^{\beta} \text {, }
\end{aligned}
$$




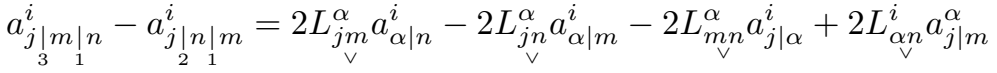

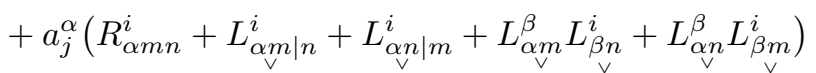

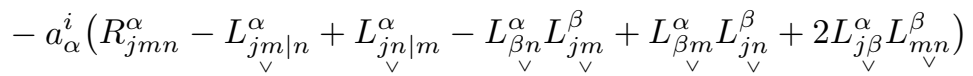

$$
\begin{aligned}
& -2 a_{\beta}^{\alpha} L_{\alpha \underset{\vee}{ }}^{i} L_{j n}^{\beta}
\end{aligned}
$$

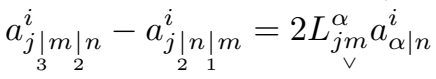

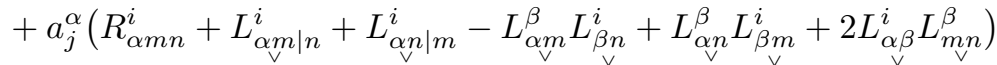

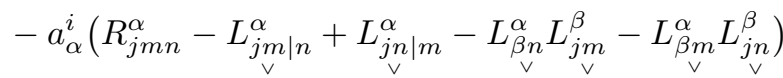

$$
\begin{aligned}
& -2 a_{\beta}^{\alpha} L_{\alpha \eta}^{i} L_{j m}^{\beta}
\end{aligned}
$$

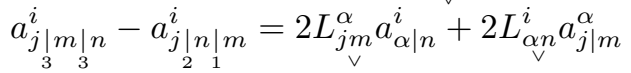

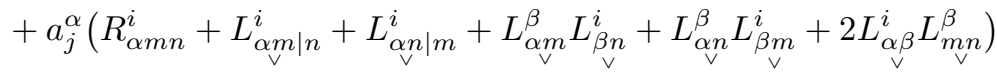

$$
\begin{aligned}
& -a_{\alpha}^{i}\left(R_{j m n}^{\alpha}-L_{j m \mid n}^{\alpha}+L_{j n \mid m}^{\alpha}-L_{\beta n}^{\alpha} L_{j m}^{\beta}-L_{\beta m}^{\alpha} L_{j n}^{\beta}\right),
\end{aligned}
$$

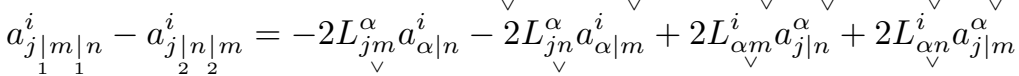

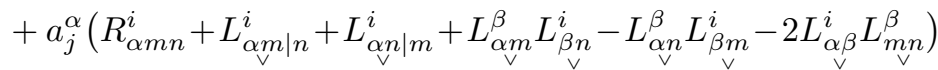

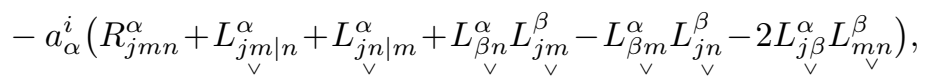

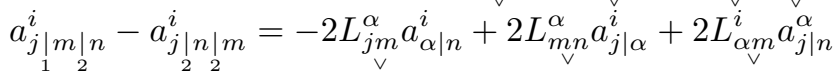

$$
\begin{aligned}
& +a_{j}^{\alpha}\left(R_{\alpha m n}^{i}+L_{\alpha m \mid n}^{i}+L_{\alpha n \mid m}^{i}-L_{\alpha{ }_{\vee}}^{\beta} L_{\beta n}^{i}-L_{\alpha n}^{\beta} L_{\beta m}^{i}\right)
\end{aligned}
$$

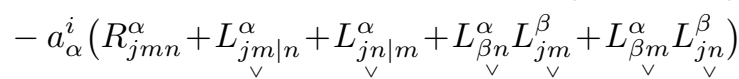

$$
\begin{aligned}
& +2 a_{\beta}^{\alpha}\left(L_{\alpha m}^{i} L_{j n}^{\beta}+L_{\alpha \vee}^{i} L_{j m}^{\beta}\right) \text {, }
\end{aligned}
$$

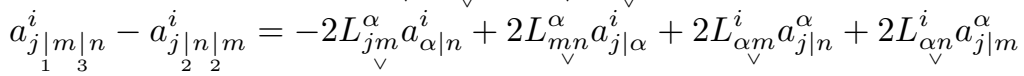

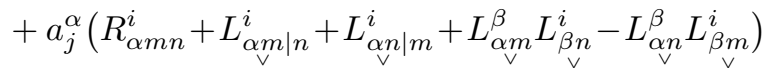

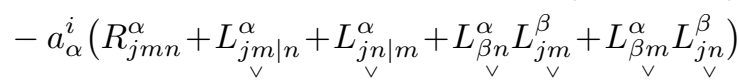

$$
\begin{aligned}
& +2 a_{\beta}^{\alpha} L_{\alpha m}^{i} L_{j n}^{\beta}
\end{aligned}
$$

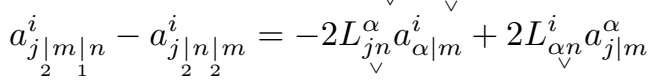

$$
\begin{aligned}
& +a_{j}^{\alpha}\left(R_{\alpha m n}^{i}-L_{\alpha m \mid n}^{i}+L_{\alpha \vee \mid m}^{i}-L_{\alpha{ }_{\vee}}^{\beta} L_{\beta n}^{i}-L_{\alpha n}^{\beta} L_{\beta m}^{i}\right)
\end{aligned}
$$

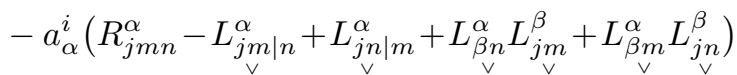

$$
\begin{aligned}
& +2 a_{\beta}^{\alpha}\left(L_{\alpha \underset{\vee}{ }}^{i} L_{j n}^{\beta}+L_{\alpha \vee}^{i} L_{j m}^{\beta}\right),
\end{aligned}
$$




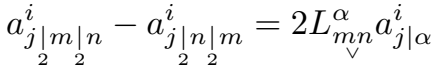

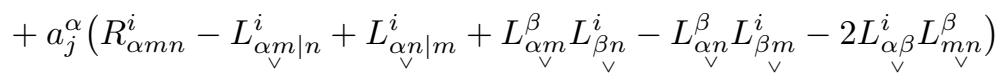

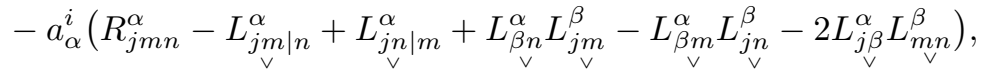

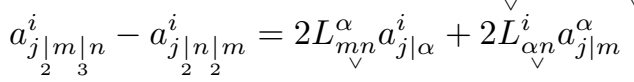

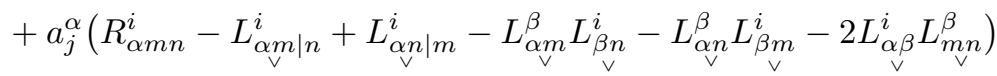

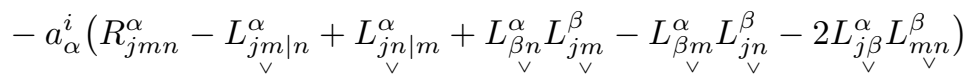

$$
\begin{aligned}
& +2 a_{\beta}^{\alpha} L_{\alpha n}^{i} L_{j m}^{\beta}
\end{aligned}
$$

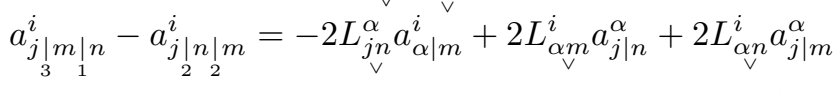

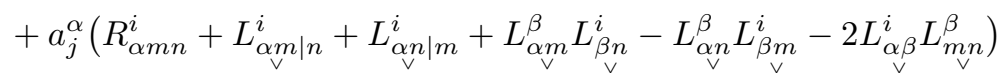

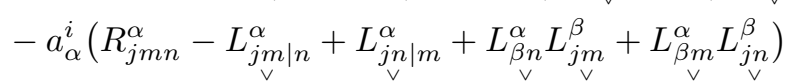

$$
\begin{aligned}
& +2 a_{\beta}^{\alpha} L_{\alpha \vee}^{i} L_{j m}^{\beta} \\
& a_{\substack{|m| m \mid n \\
3}}^{i}-a_{\substack{\left|{ }_{2}\right| n \mid m \\
i}}^{i}=2 L_{\underset{\vee}{m} n}^{\alpha} a_{j \mid \alpha}^{i}+2 L_{\underset{\vee}{ }}^{i} a_{j \mid n}^{\alpha}
\end{aligned}
$$

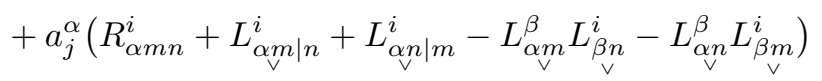

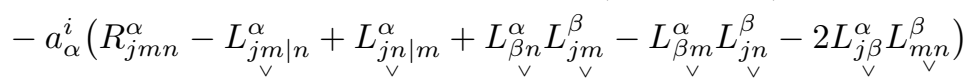

$$
\begin{aligned}
& +2 a_{\beta}^{\alpha} L_{\alpha m}^{i} L_{j n}^{\beta}
\end{aligned}
$$

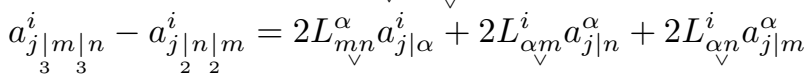

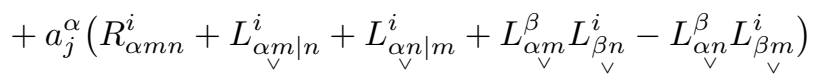

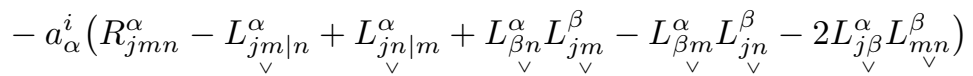

$$
\begin{aligned}
& +2 a_{\beta}^{\alpha}\left(L_{\alpha \vee}^{i} L_{j m}^{\beta}+L_{\alpha \underset{\vee}{ }}^{i} L_{j n}^{\beta}\right) \text {, }
\end{aligned}
$$

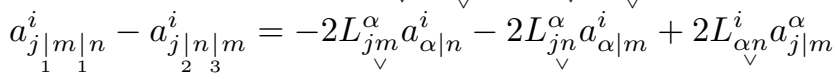

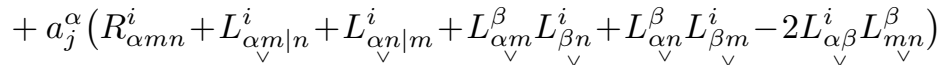

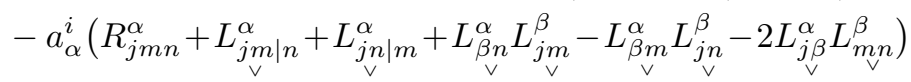

$$
\begin{aligned}
& -2 a_{\beta}^{\alpha} L_{\alpha \mathrm{v}}^{i} L_{j n}^{\beta}
\end{aligned}
$$

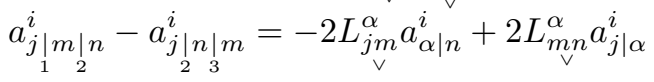

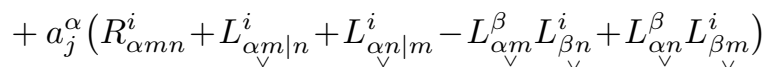

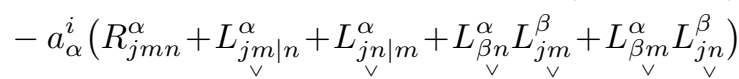

$$
\begin{aligned}
& +2 a_{\beta}^{\alpha} L_{\underset{\vee}{\alpha n}}^{i} L_{\substack{v m \\
\beta}}^{\beta}
\end{aligned}
$$




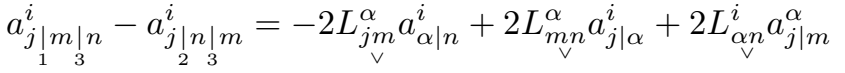

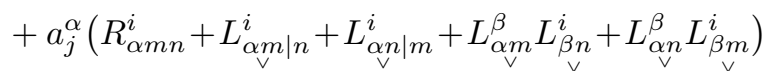

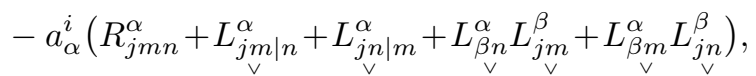

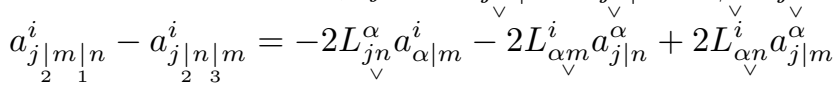

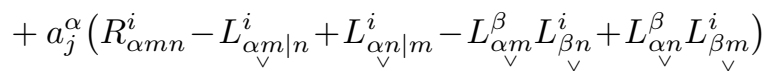

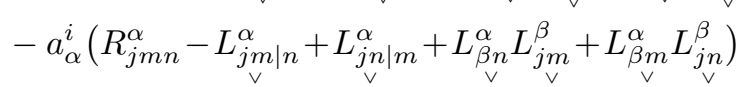

$$
\begin{aligned}
& +2 a_{\beta}^{\alpha} L_{\alpha \vee}^{i} L_{j m}^{\beta}, \\
& a_{\substack{|m| m \mid n \\
i}}^{i}-a_{\substack{\left|{ }_{2}\right| n \mid m \\
i}}^{i}=2 L_{\underset{\vee}{m} n}^{\alpha} a_{j \mid \alpha}^{i}-2 L_{\underset{\vee}{\alpha}}^{i} a_{j \mid n}^{\alpha}
\end{aligned}
$$

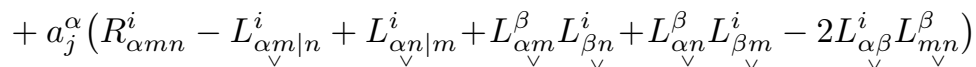

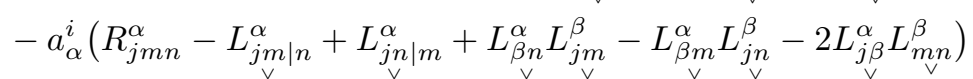

$$
\begin{aligned}
& -2 a_{\beta}^{\alpha} L_{\alpha m}^{i} L_{j n}^{\beta}
\end{aligned}
$$

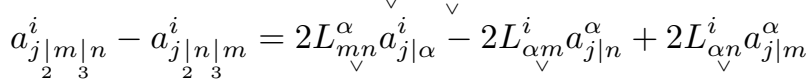

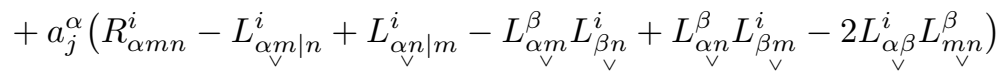

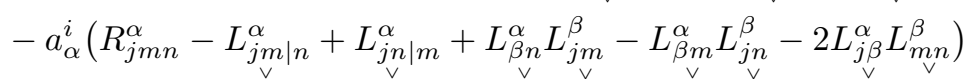

$$
\begin{aligned}
& -2 a_{\beta}^{\alpha}\left(L_{\alpha m}^{i} L_{j n}^{\beta}-L_{\alpha n}^{i} L_{j m}^{\beta}\right) \text {, }
\end{aligned}
$$

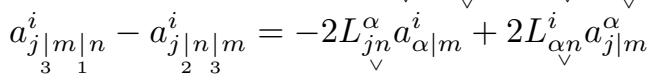

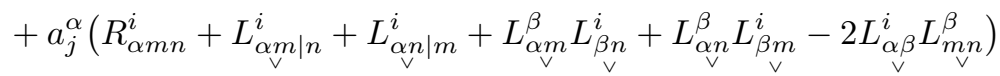

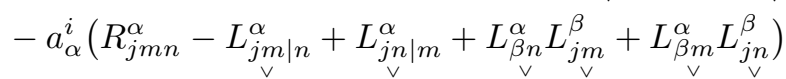

$$
\begin{aligned}
& -2 a_{\beta}^{\alpha}\left(L_{\alpha \underset{\vee}{ }}^{i} L_{j n}^{\beta}-L_{\underset{\vee}{ }}^{i} L_{j m}^{\beta}\right),
\end{aligned}
$$

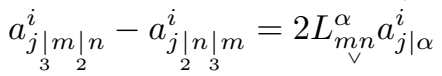

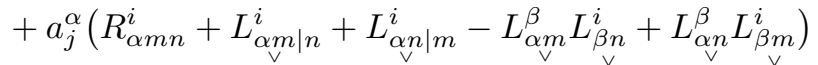

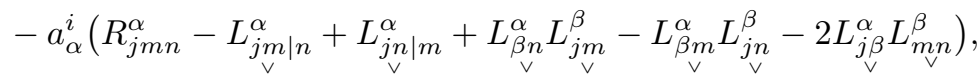

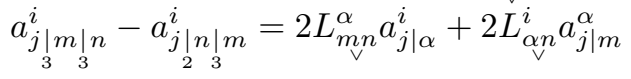

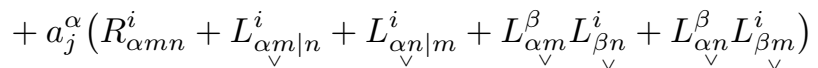

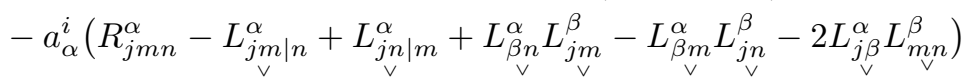

$$
\begin{aligned}
& +2 a_{\beta}^{\alpha} L_{\alpha \bigvee}^{i} L_{j m}^{\beta}
\end{aligned}
$$

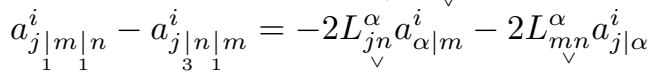

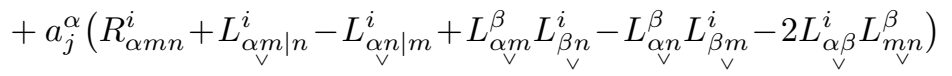

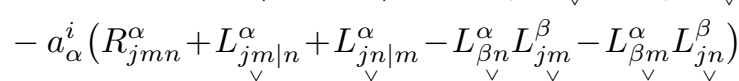

$$
\begin{aligned}
& -2 a_{\beta}^{\alpha} L_{\alpha \underset{\vee}{ }}^{i} L_{j n}^{\beta}
\end{aligned}
$$




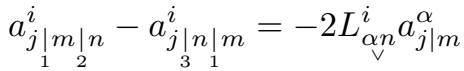

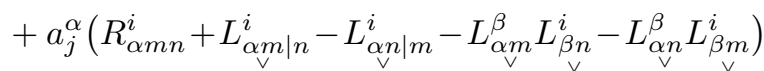

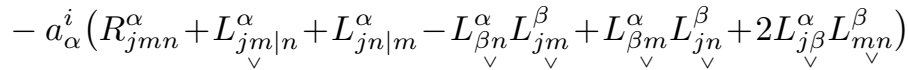

$$
\begin{aligned}
& +2 a_{\beta}^{\alpha} L_{\alpha n}^{i} L_{j m}^{\beta}
\end{aligned}
$$

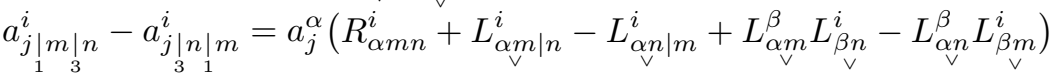

$$
\begin{aligned}
& -a_{\alpha}^{i}\left(R_{j m n}^{\alpha}+L_{j m \mid n}^{\alpha}+L_{j n \mid m}^{\alpha}-L_{\beta n}^{\alpha} L_{j m}^{\beta}+L_{\beta m}^{\alpha} L_{j n}^{\beta}+2 L_{j \beta}^{\alpha} L_{m n}^{\beta}\right) \text {, }
\end{aligned}
$$

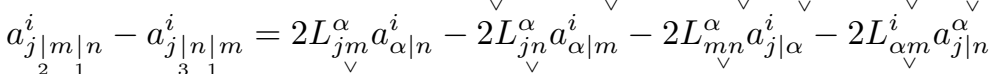

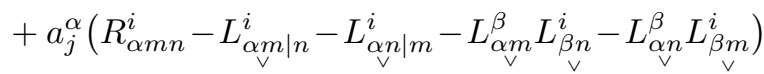

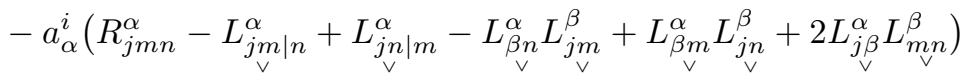

$$
\begin{aligned}
& +2 a_{\beta}^{\alpha} L_{\alpha n}^{i} L_{j m}^{\beta}
\end{aligned}
$$

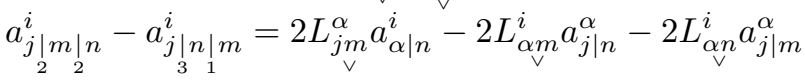

$$
\begin{aligned}
& +a_{j}^{\alpha}\left(R_{\alpha m n}^{i}-L_{\alpha \vee \mid n}^{i}-L_{\alpha \vee \mid m}^{i}+L_{\alpha m}^{\beta} L_{\beta n}^{i}-L_{\alpha \vee}^{\beta} L_{\beta m}^{i}-2 L_{\alpha \beta}^{i} L_{m_{\vee} n}^{\beta}\right)
\end{aligned}
$$

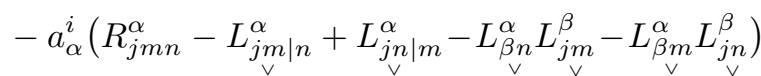

$$
\begin{aligned}
& -2 a_{\beta}^{\alpha} L_{\alpha \mathrm{v}}^{i} L_{j n}^{\beta} \text {, } \\
& a_{\substack{|m| m \mid n \\
i}}^{i}-a_{\substack{j|n| m \\
3}}^{i}=2 L_{\substack{j m \\
\vee}}^{\alpha} a_{\alpha \mid n}^{i}-2 L_{\underset{\vee}{\alpha m}}^{i} a_{j \mid n}^{\alpha}
\end{aligned}
$$

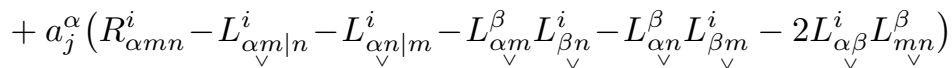

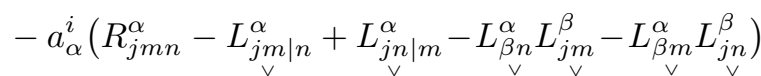

$$
\begin{aligned}
& -2 a_{\beta}^{\alpha}\left(L_{\alpha m}^{i} L_{j n}^{\beta}-L_{\alpha \vee}^{i} L_{j m}^{\beta}\right) \text {, }
\end{aligned}
$$

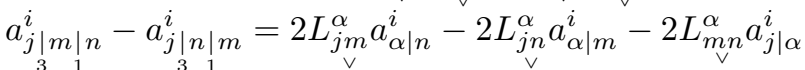

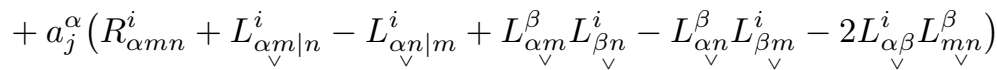

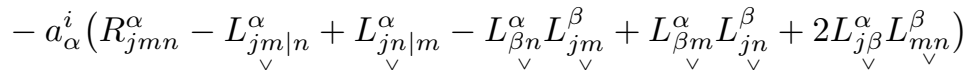

$$
\begin{aligned}
& -2 a_{\beta}^{\alpha}\left(L_{\alpha m}^{i} L_{j n}^{\beta}-L_{\alpha n}^{i} L_{j m}^{\beta}\right) \text {, }
\end{aligned}
$$

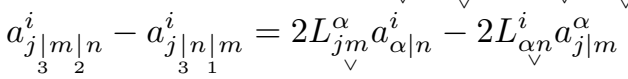

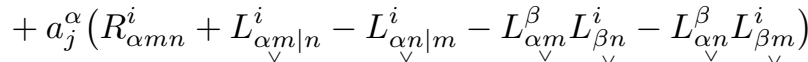

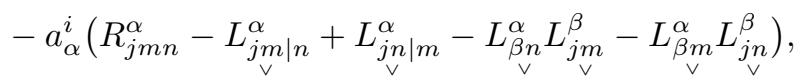




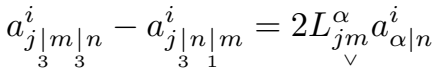

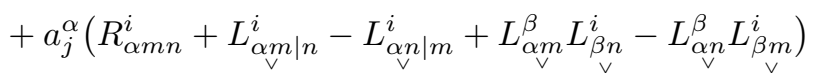

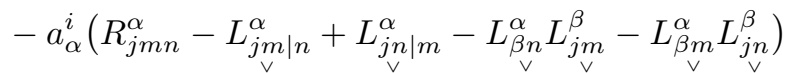

$$
\begin{aligned}
& +2 a_{\beta}^{\alpha} L_{\alpha n}^{i} L_{j m}^{\beta}
\end{aligned}
$$

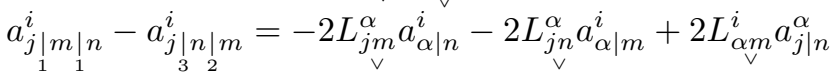

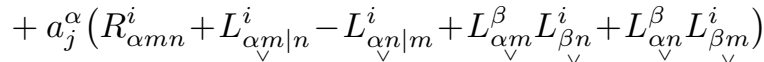

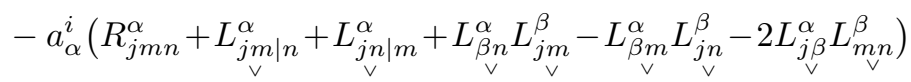

$$
\begin{aligned}
& -2 a_{\beta}^{\alpha} L_{\alpha n}^{i} L_{j m}^{\beta}
\end{aligned}
$$

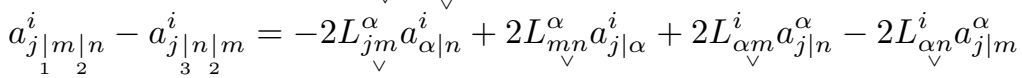

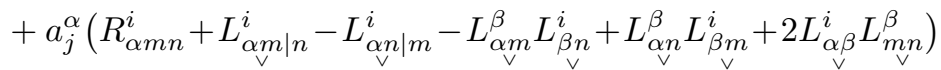

$$
\begin{aligned}
& -a_{\alpha}^{i}\left(R_{j m n}^{\alpha}+L_{j m \mid n}^{\alpha}+L_{j n \mid m}^{\alpha}+L_{\beta n}^{\alpha} L_{j m}^{\beta}+L_{\beta m}^{\alpha} L_{j n}^{\beta}\right) \\
& +2 a_{\beta}^{\alpha} L_{\alpha m}^{i} L_{j n}^{\beta}
\end{aligned}
$$

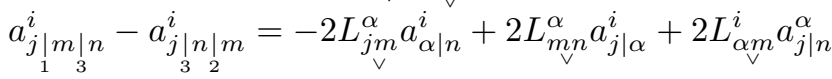

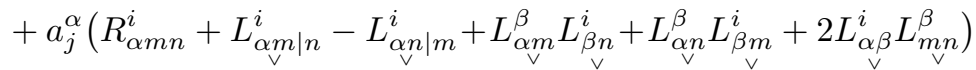

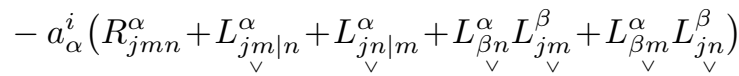

$$
\begin{aligned}
& +2 a_{\beta}^{\alpha}\left(L_{\alpha m}^{i} L_{j n}^{\beta}-L_{\alpha \vee}^{i} L_{j m}^{\beta}\right) \text {, }
\end{aligned}
$$

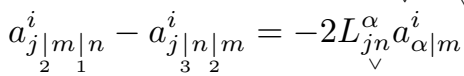

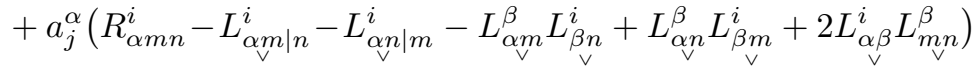

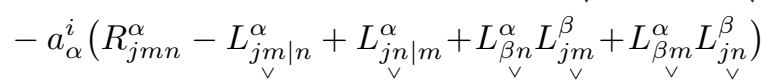

$$
\begin{aligned}
& +2 a_{\beta}^{\alpha} L_{\alpha \mathrm{V}}^{i} L_{j n}^{\beta}
\end{aligned}
$$

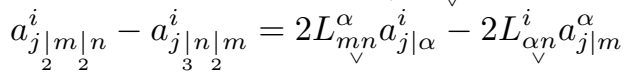

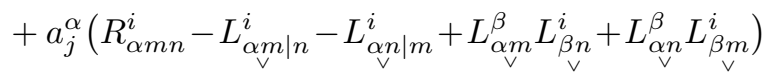

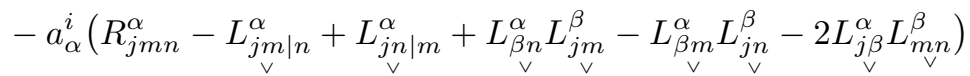

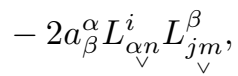




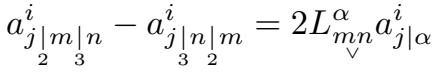

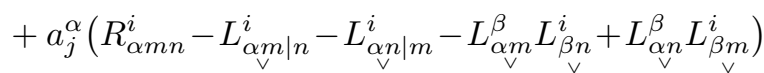

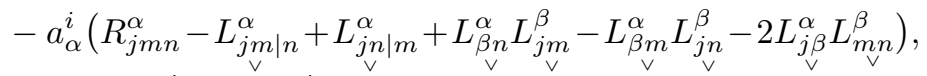

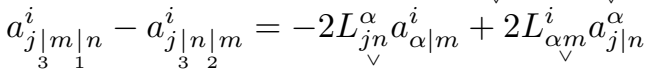

$$
\begin{aligned}
& +a_{j}^{\alpha}\left(R_{\alpha m n}^{i}+L_{\underset{\vee}{\alpha m \mid n}}^{i}-L_{\underset{\vee}{\alpha n \mid m}}^{i}+L_{\underset{\vee}{\alpha m}}^{\beta} L_{\beta n}^{i}+L_{\underset{\vee}{\alpha n}}^{\beta} L_{\beta m}^{i}\right) \\
& -a_{\alpha}^{i}\left(R_{j m n}^{\alpha}-L_{j m \mid n}^{\alpha}+L_{j n \mid m}^{\alpha}+L_{\beta n}^{\alpha} L_{j m}^{\beta}+L_{\beta m}^{\alpha} L_{j n}^{\beta}\right),
\end{aligned}
$$

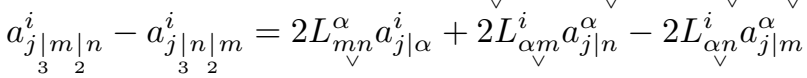

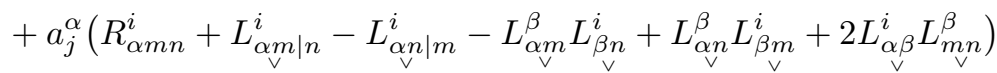

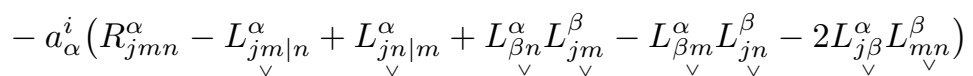

$$
\begin{aligned}
& +2 a_{\beta}^{\alpha}\left(L_{\alpha m}^{i} L_{j n}^{\beta}-L_{\alpha n}^{i} L_{j m}^{\beta}\right) \text {, }
\end{aligned}
$$

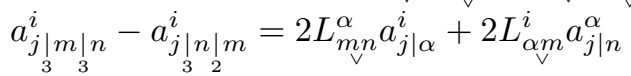

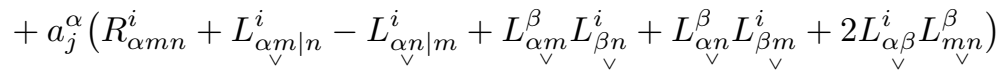

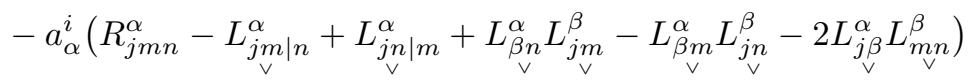

$$
\begin{aligned}
& +2 a_{\beta}^{\alpha} L_{\alpha \mathrm{v}}^{i} L_{j n}^{\beta}
\end{aligned}
$$

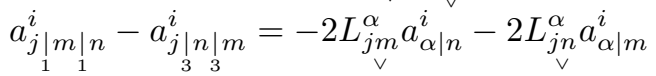

$$
\begin{aligned}
& +a_{j}^{\alpha}\left(R_{\alpha m n}^{i}+L_{\alpha \mathrm{V} \mid n}^{i}-L_{\alpha n \mid m}^{i}+L_{\alpha \mathrm{V}}^{\beta} L_{\beta n}^{i}-L_{\alpha \vee}^{\beta} L_{\beta m}^{i}\right)
\end{aligned}
$$

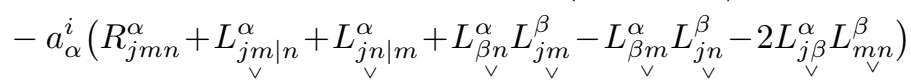

$$
\begin{aligned}
& -2 a_{\beta}^{\alpha}\left(L_{\alpha n}^{i} L_{j m}^{\beta}+L_{\alpha m}^{i} L_{j n}^{\beta}\right),
\end{aligned}
$$

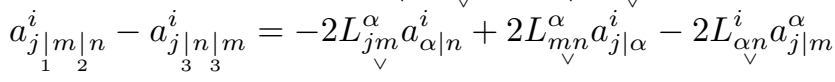

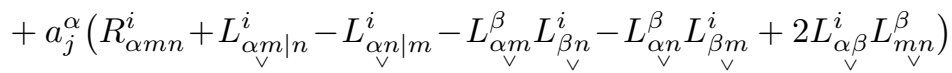

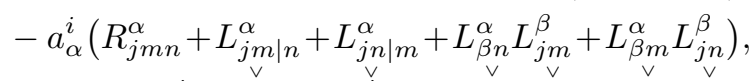

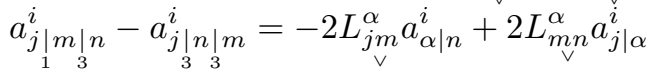

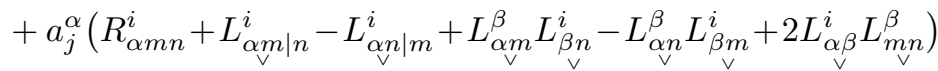

$$
\begin{aligned}
& -a_{\alpha}^{i}\left(R_{j m n}^{\alpha}+L_{j m \mid n}^{\alpha}+L_{j n \mid m}^{\alpha}+L_{\beta n}^{\alpha} L_{j m}^{\beta}+L_{\beta m}^{\alpha} L_{j n}^{\beta}\right) \\
& -2 a_{\beta}^{\alpha} L_{\alpha \bigvee}^{i} L_{j m}^{\beta}
\end{aligned}
$$

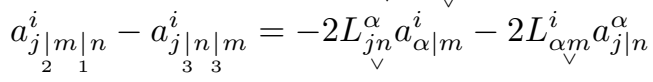

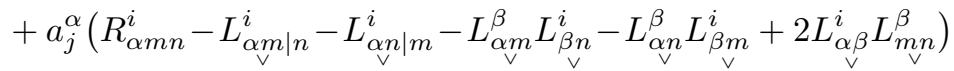

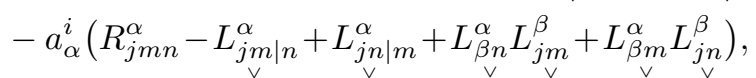




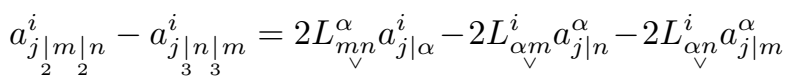

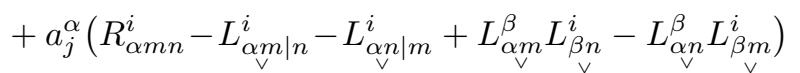

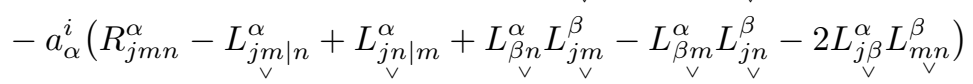

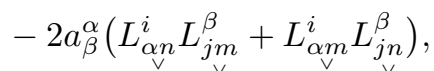

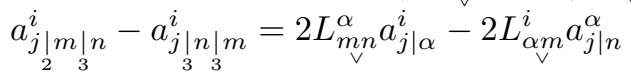

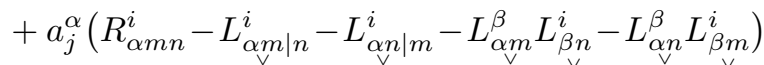

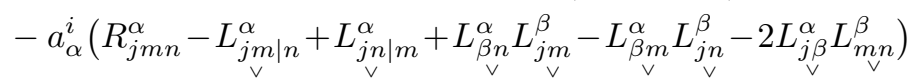

$$
\begin{aligned}
& -2 a_{\beta}^{\alpha} L_{\alpha \mathrm{v}}^{i} L_{j n}^{\beta}
\end{aligned}
$$

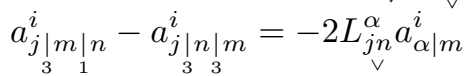

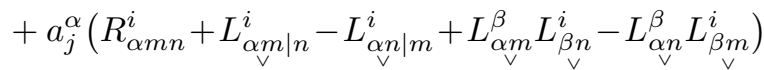

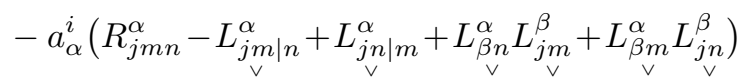

$$
\begin{aligned}
& -2 a_{\beta}^{\alpha} L_{\alpha m}^{i} L_{j n}^{\beta}
\end{aligned}
$$

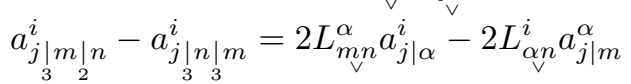

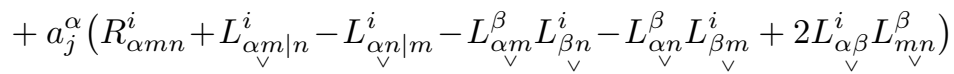

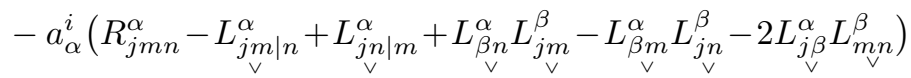

$$
\begin{aligned}
& -2 a_{\beta}^{\alpha} L_{\underset{\vee}{\alpha n}}^{i} L_{j m}^{\beta}
\end{aligned}
$$

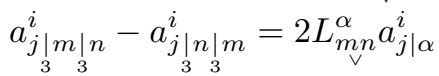

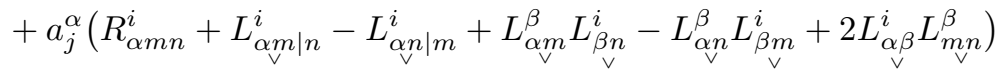

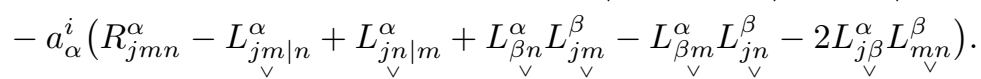

\section{Conclusion}

This manuscript conducted the research of the components of curvatures for the non-symmetric affine connection space $\mathbb{G}_{N}$ with respect to three of four plus one kinds of covariant derivatives $(1.2,1.3)$.

Here, it was elaborated that curvature pseudotensors are not components of the differences $a_{j \mid m}^{i} \underset{v_{1} \mid}{\mid} n-a_{w_{1}}^{i} \underset{v_{2}\left|{ }_{w_{2}}\right| m}{ }, v_{1}, v_{2}, w_{1}, w_{2} \in\{0,1,2,3,4\}$.

In future work, we will study the commutation formulae obtained with respect to all triples of linearly independent geometrical objects $a_{j \mid k}^{i}, p=0, \ldots, 4$. 


\section{REF E R E N C E S}

1. L. P. Eisenhart: Non-Riemannian Geometry, New York, 1927.

2. J. Mikeš, E. Stepanova, A. Vanžurova, et al.: Differential geometry of special mappings, Olomouc: Palacky University, 2015.

3. S. M. Minčić, Ricci identities in the space of non-symmetric affine connexion, Mat. Vesnik, 10 (25) sv. 2, (1973), 161-172.

4. S. M. Minčić: Curvature tensors of the space of non-symmetric affine connexion, obtained from the curvature pseudotensors, Matematički Vesnik, 13 (28) (1976), 421-435.

5. S. M. Minčić, New commutation formulas in the non-symmetric affine connexion space, Publ. Inst. Math., Nouv. Sér. 22 (1977) 189-199.

6. S. M. Minčić: Independent curvature tensors and pseudotensors of spaces with nonsymmetric affine connexion, Coll. Math. Soc. János Bolayai, 31. Dif. geom., Budapest (Hungary), (1979), 445-460.

7. S. M. Minčić: On Ricci Type Identities in Manifolds With Non-Symmetric Affine Connection, Publications De L'Institut Mathématique, Nouvelle série, tome 94 (108) (2013), 205-217.

8. S. M. Minčić and Lj. S. Velimirović: Spaces With Non-Symmetric Affine Connection, Novi Sad J. Math., Vol. 38, No. 3, 2008, 157-164.

9. M. Z. Petrović, Generalized para-Kähler Spaces in Eisenharts Sense Admitting a Holomorphically Projective Mapping, Filomat, Vol. 33, No. 13, 2019, 4001-4012.

10. M. Z. Petrović, Lj. S. Velimirović, Generalized Kähler spaces in Eisenhart's sense admitting a holomorphically projective mapping, Mediterr. J. Math. (2018) 15:150.

11. M. Z. Petrović, Lj. S. Velimirović, A new type of generalized para-Kahler spaces and holomorphically projective transformations, Bull. Iran. Math. Soc., Vol. 45, No. 4, 2019, 1021-1043.

12. N. S. Sinyukov, Geodesic mappings of Riemannian spaces, (in Russian), "Nauka", Moscow, 1979.

13. M. S. Stanković, M. Lj. Zlatanović, Lj. S. Velimirović, Equitorsion holomorphically projective mappings of generalized Kählerian space of the first kind, Czechoslovak Mathematical Journal, Vol. 60, (2010) 635-653.

14. M. Lj. Zlatanović, New projective tensors for equitorsion geodesic mappings, Applied Mathematics Letters, Vol. 25, No. 5, 2012, 890-897.

Nenad O. Vesić

Mathematical Institute of Serbian Academy of Sciences and Arts

Kneza Mihaila 36

11000 Belgrade, Serbia

n.o.vesic@outlook.com 